\title{
Topological Indices Study of Molecular Structure in Anticancer Drugs
}

\author{
Wei Gao, ${ }^{1}$ Weifan Wang, ${ }^{2}$ and Mohammad Reza Farahani ${ }^{3}$ \\ ${ }^{1}$ School of Information Science and Technology, Yunnan Normal University, Kunming 650500, China \\ ${ }^{2}$ Department of Mathematics, Zhejiang Normal University, Jinhua 321004, China \\ ${ }^{3}$ Department of Applied Mathematics, Iran University of Science and Technology, Narmak, Tehran 16844, Iran
}

Correspondence should be addressed to Wei Gao; gaowei@ynnu.edu.cn

Received 26 December 2015; Accepted 15 February 2016

Academic Editor: José L. A. Mediano

Copyright (c) 2016 Wei Gao et al. This is an open access article distributed under the Creative Commons Attribution License, which permits unrestricted use, distribution, and reproduction in any medium, provided the original work is properly cited.

\begin{abstract}
Numerous studies indicate that there is strong inherent relationship between the chemical characteristics of chemical compounds and drugs (e.g., boiling point and melting point) and their molecular structures. Topological indices defined on these chemical molecular structures can help researchers better understand the physical features, chemical reactivity, and biological activity. Thus, the study of the topological indices on chemical structure of chemical materials and drugs can make up for lack of chemical experiments and can provide a theoretical basis for the manufacturing of drugs and chemical materials. In this paper, we focus on the family of smart polymer which is widely used in anticancer drugs manufacturing. Several topological indices are determined in view of edge dividing methods, and these results remedy the lack of chemical and medicine experiments thus providing the theoretical basis for pharmaceutical engineering.
\end{abstract}

\section{Introduction}

In this era of rapid technological development, chemical and pharmaceutical techniques in recent years have been rapidly evolved, and thus a large number of new nanomaterials, crystalline materials, and drugs emerge every year. To determine the chemical properties of such a large number of new compounds and new drugs requires a large amount of chemical experiments, thereby greatly increasing the workload of the chemical and pharmaceutical researchers. Fortunately, the chemical based experiments found that there was strong connection between topology molecular structures and their physical behaviors, chemical characteristics, and biological features, such as melting point, boiling point, and toxicity of drugs (see Wiener [1] and Katritzky et al. [2] as examples).

The topological index of a molecule structure can be considered as a nonempirical numerical quantity which quantitates the molecular structure and its branching pattern. In this point of view, topological index can be regarded as a score function which maps each molecular structure to a real number and used as a descriptor of the molecule under testing. There are several famous indices applied in chemical engineering (e.g., QSPR/QSAR study) for grasping the relationships between the molecular structure and the potential physicochemical characteristics, such as PI index, Zagreb index, harmonic index, Wiener index, and connectivity index (see Yan et al. [3], Gao and Shi [4], and Gao and Wang [5, 6] for more details).

In theoretical chemistry setting, chemical compounds, materials, and drugs are expressed as (molecular) graphs in which each vertex represents an atom of molecule structure and each edge implies covalent bounds between two atoms. Let $G=(V(G), E(G))$ be a (molecular) graph with vertex set $V(G)$ and edge set $E(G)$, respectively. We assume that all the graphs considered in this paper are simple graphs, that is, no loop and multiple edge. The notations and terminologies used but not clearly undefined in this paper can be found in [7].

There are several degree based indices introduced to test the properties of compounds and drugs, which have been widely used in chemical and pharmacy engineering. Bollobas 
and Erdos [8] introduced the general Randic index stated as

$$
R_{k}(G)=\sum_{e=u v}(d(u) d(v))^{k},
$$

where $k$ is a real number and $d(u)$ is the degree of vertex $u$. Li and Liu [9] studied the first three minimum general Randic indices of tree structures, and the corresponding extremal trees are described. Liu and Gutman [10] estimated the general Randic index and its special situations, the ordinary index and modified Zagreb index. In what follows, we always assume that $k$ is a real number.

By setting $k=1$ and $k=-1$, formula (1) becomes the second Zagreb index $\left(M_{2}(G)\right)$ and the modified second Zagreb index $\left(M_{2}^{*}(G)\right)$ which are stated as

$$
\begin{aligned}
& M_{2}(G)=\sum_{e=u v} d(u) d(v), \\
& M_{2}^{*}(G)=\sum_{e=u v} \frac{1}{d(u) d(v)},
\end{aligned}
$$

respectively.

The sum connectivity index $(\chi(G))$ of molecular graph $G$ was defined by

$$
\chi(G)=\sum_{e=u v}(d(u)+d(v))^{-1 / 2} .
$$

Few years ago, Zhou and Trinajstic [11] extended this concept and introduced the general sum connectivity as follows:

$$
\chi_{k}(G)=\sum_{e=u v}(d(u)+d(v))^{k} .
$$

Note that Shirdel et al. [12] introduced a new version of Zagreb indices named Hyper-Zagreb index as

$$
H M(G)=\sum_{e=u v}(d(u)+d(v))^{2} .
$$

Obviously, Hyper-Zagreb index is just a special case of general sum connectivity when $k=2$.

The harmonic index for a molecular graph $G$ is defined as

$$
H(G)=\sum_{e=u v} \frac{2}{d(u)+d(v)} .
$$

Favaron et al. [13] researched the relationship between the eigenvalues of molecular graphs and harmonic index. Zhong [14] obtained the minimum and maximum values of the harmonic index for connected molecular structures and trees, and the corresponding extremal molecular graphs are described. Wu et al. [15] yielded the minimum harmonic index of molecular graphs with $\delta(G) \geq 2$. Liu [16] gave several relations between the harmonic index and diameter of molecular graphs.

Very recently, in order to extend harmonic index for more chemical engineering applications, Yan et al. [3] introduced the general version of harmonic index which was formulated by

$$
H_{k}(G)=\sum_{e=u v}\left(\frac{2}{d(u)+d(v)}\right)^{k}
$$

Eliasi and Iranmanesh [17] proposed the ordinary geometric-arithmetic index as the extension of geometricarithmetic index which was stated as

$$
\operatorname{OGA}_{k}(G)=\sum_{e=u v}\left(\frac{2 \sqrt{d(u) d(v)}}{d(u)+d(v)}\right)^{k} .
$$

Azari and Iranmanesh [18] raised the generalized version of Zagreb index as

$$
M_{t_{1}, t_{2}}(G)=\sum_{e=u v}\left(d(u)^{t_{1}} d(v)^{t_{2}}+d(u)^{t_{2}} d(v)^{t_{1}}\right),
$$

where parameters $t_{1}$ and $t_{2}$ are arbitrary nonnegative integers.

Several polynomials closely related to degree based indices are also introduced. For example, the first and the second Zagreb polynomials corresponding to first and the second Zagreb indices are expressed as

$$
\begin{aligned}
& M_{1}(G, x)=\sum_{e=u v} x^{d(u)+d(v)}, \\
& M_{2}(G, x)=\sum_{e=u v} x^{d(u) d(v)},
\end{aligned}
$$

respectively.

Additionally, the third Zagreb index and third Zagreb polynomial were defined as

$$
\begin{aligned}
M_{3}(G) & =\sum_{e=u v}|d(u)-d(v)|, \\
M_{3}(G, x) & =\sum_{e=u v} x^{|d(u)-d(v)|} .
\end{aligned}
$$

As degree based topological indices, the multiplicative version of these Zagreb indices (the first multiplicative Zagreb index $P M_{1}(G)$ and the second multiplicative Zagreb index $\mathrm{PM}_{2}(G)$ ) of a (molecular) graph $G$ were introduced by Gutman [19] and Ghorbani and Azimi [20] as

$$
\begin{aligned}
& P M_{1}(G)=\prod_{e=u v \in E(G)}(d(u)+d(v)), \\
& P M_{2}(G)=\prod_{e=u v \in E(G)}(d(u) d(v)) .
\end{aligned}
$$

Several advances on $P M_{1}(G)$ and $P M_{2}(G)$ can be referred to Eliasi et al. [21], Xu and Das [22], and Farahani [23].

Furthermore, the redefined version of Zagreb indices of a (molecular) graph $G$ was introduced by Ranjini et al. [24] as follows:

$$
\begin{aligned}
& \operatorname{ReZG}_{1}(G)=\sum_{e=u v \in E(G)} \frac{d(u)+d(v)}{d(u) d(v)}, \\
& \operatorname{ReZG}_{2}(G)=\sum_{e=u v \in E(G)} \frac{d(u) d(v)}{d(u)+d(v)}, \\
& \operatorname{ReZG}_{3}(G)=\sum_{e=u v \in E(G)}(d(u) d(v))(d(u)+d(v)) .
\end{aligned}
$$

Here, $Z G_{1}(G), Z G_{2}(G)$, and $Z G_{3}(G)$ are first, second, and third redefined Zagreb indices. 
<smiles>COc1cccc2c1C(=O)C1C(O)=C3CC(O)(C(=O)CO)CC(OC4CC(NC(=O)CC(NC(C)C)C(=O)NCCCC(C)OCCC(C)(C)OC)C(O)C(C)O4)C3=C(O)C1C2=O</smiles>

FIGURE 1: Dox-loaded micelle comprising PEG-PAsp block copolymer with chemically conjugated Dox $S P[n]$.

In the following contents, we first introduce the smart polymers of Dox-loaded micelle comprising PEG-PAsp block copolymer with chemically conjugated Dox and explain the importance of this molecular structure. This is the reason why we focus on the topological indices computation on this family of molecular graphs. Then, in Section 3, we present our main results and detailed proofs.

\section{Motivation}

As a special class of macromolecules, smart polymers manifest impressive response to physiochemical change if their circumstance has slight interference, for example, changes on $\mathrm{PH}$ value, ionic disturbance, magnetic field, light, and temperature (see Hai and Broekmann [25], do Nascimento Marques et al. [26], and Kroning et al. [27]). Thus, smart polymers are also denoted by environmentally responsive systems or stimuli responsive ones. As good delivery systems, these structures have wide applications in biomedical field, for instance, smart polymers with nucleic acid or protein delivery to intracellular targets just like nucleus or ribosome in tissue engineering (see Chonkar et al. [28], Hrubý et al. [29], Duro-Castano et al. [30], and Khandare and Calderón [31]). As a special class of smart polymer, polymeric micelles (e.g., Dox-conjugated PEG-b-poly(aspartate) (PEG-PAsp) block copolymers; see Shanthi et al. [32] and Osada et al. [33]) are widely applied in delivering anticancer drug.

The Dox-loaded micelle containing PEG-PAsp block copolymer with chemically conjugated Dox (see Figure 1 for its detailed structure) is a famous family of smart polymer which is used as anthracycline anticancer antibiotic and applied in treating various kinds of cancers. It is employed as excipients and drug delivery carriers for strengthening the stability and the times of drug retention. Thus, it has powerful anticancer activity and is widely considered in the pharmacy field (see Nishiyama and Kataoka [34] and Butt et al. [35] for more details).<smiles>COCCOCCCNC(=O)C(N)CC(=O)NC1CC(OC2CC(O)(C(=O)CO)CC3=C2C(O)=C2C(=O)c4cccc(OC)c4C(=O)C2C3O)OC(C)C1O</smiles>

FIGURE 2: The molecular structure of $S P[1]$.

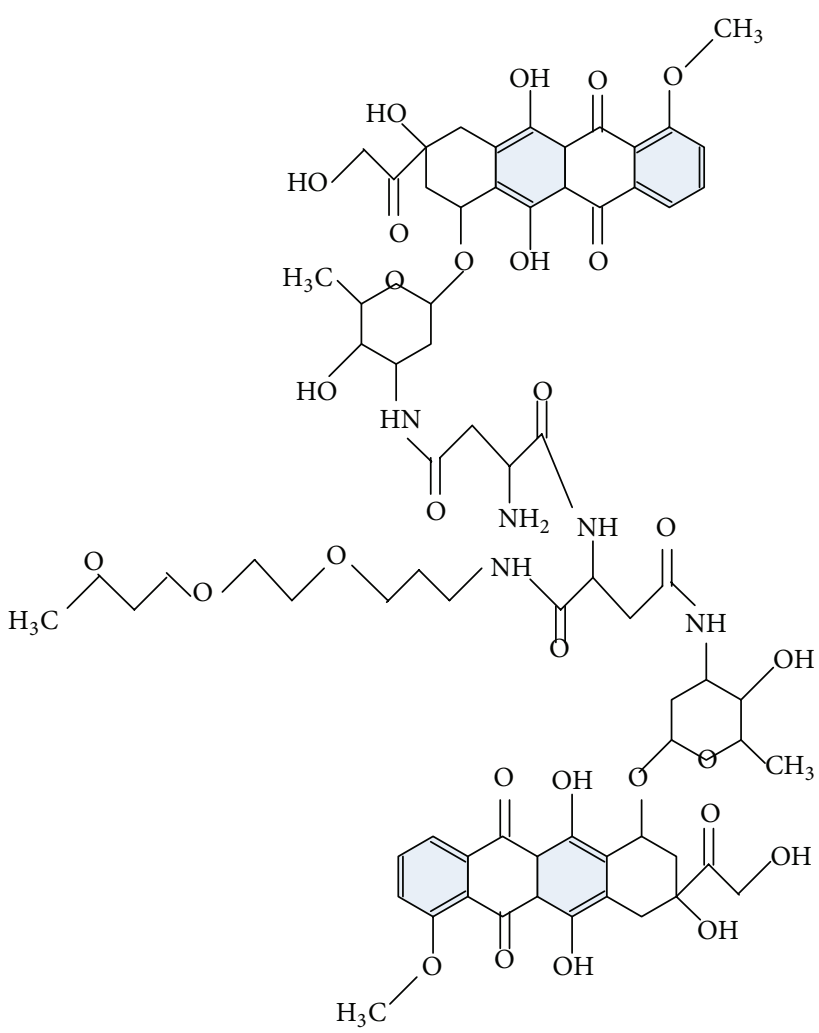

FIgURE 3: The molecular structure of $S P$ [2].

As can be seen in Figure 1, the integer number $n$ is step of growth in this kind of polymers. We depict the structure of Dox-loaded micelle containing PEG-PAsp block copolymer with chemically conjugated Dox when $n=1,2$, and 3 (see Figures 2, 3, and 4, resp.).

Although several advances have been made in PI index, Zagreb index, Wiener index, hyper-Wiener index, and sum connectivity index of different kinds of molecular graphs, the study of topological indices for Dox-loaded micelle comprising PEG-PAsp block copolymer with chemically conjugated Dox has been largely limited. In addition, this kind of smart polymer structures is widely used in medical 


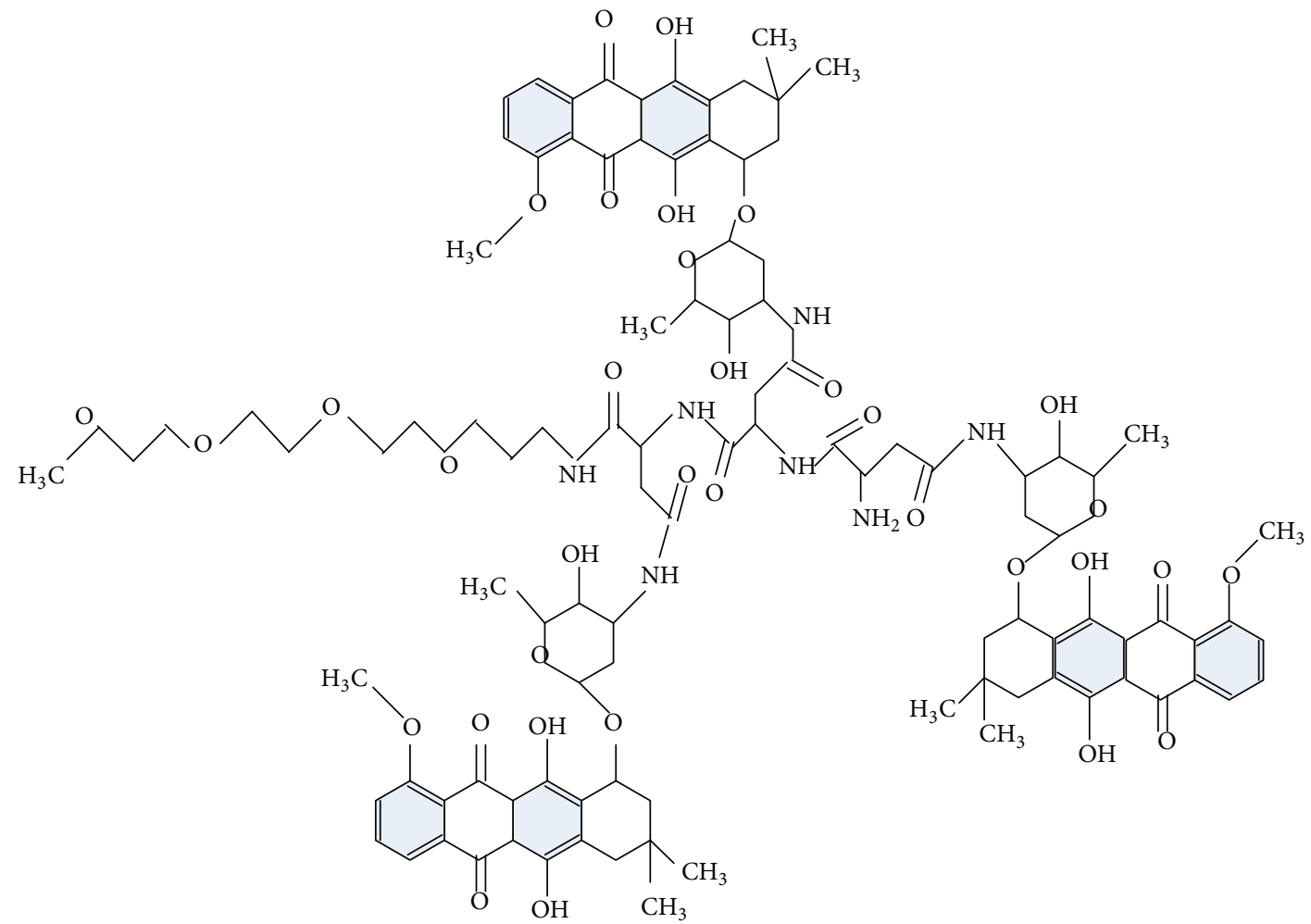

FIgURE 4: The molecular structure of $S P[3]$.

science and pharmaceutical field. For example, it plays a key role in delivery system and in the development of anticancer drugs. Based on these reasons, industrial interest and tremendous academic interest have been attracted to research the topological indices of this molecular structure from a mathematical point of view.

\section{Main Results and Proofs}

In this section, we present the main results of this paper. The techniques we used here to get the main conclusion are followed by the tricks of edge dividing.

Theorem 1. Let $S P[n]$ be the Dox-loaded micelle comprising PEG-PAsp block copolymer with chemically conjugated Dox. One has

$$
\begin{aligned}
\chi_{k}(S P[n])= & (2 n+1) 3^{k}+(14 n+2) 4^{k} \\
& +(19 n-1) 5^{k}+18 n \cdot 6^{k}+n \cdot 7^{k}, \\
R_{k}(S P[n])= & (2 n+1) 2^{k}+(9 n+1) 3^{k}+(6 n+4) 4^{k} \\
& +(18 n-1) 6^{k}+2 n \cdot 8^{k}+16 n \cdot 9^{k} \\
& +n \cdot 12^{k} .
\end{aligned}
$$

Proof. Let $\delta$ and $\Delta$ be the minimum degree and maximum degree of $S P[n]$, respectively. In what follows, we assume that the edge set $E(S P[n])$ can be divided into several partitions: (i) for any $i, 2 \delta(G) \leq i \leq 2 \Delta(G)$, let $E_{i}=\{e=u v \in E(G) \mid$ $d(u)+d(v)=i\}$

(ii) for any $j,(\delta)^{2} \leq j \leq(\Delta)^{2}$, let $E_{j}^{*}=\{e=u v \in E(G) \mid$ $d(u) d(v)=j\}$.

Specifically, by observing and computing, the edge set of $S P[n]$ can be divided into the following edge subsets:

(i) $E_{3}\left(\right.$ or $\left.E_{2}^{*}\right): d(u)=1$ and $d(v)=2$;

(ii) $E_{3}^{*}: d(u)=1$ and $d(v)=3$;

(iii) $E_{5} \cap E_{4}^{*}: d(u)=1$ and $d(v)=4$;

(iv) $E_{4} \cap E_{4}^{*}: d(u)=2$ and $d(v)=2$;

(v) $E_{6}^{*}: d(u)=2$ and $d(v)=3$;

(vi) $E_{8}^{*}: d(u)=2$ and $d(v)=4$;

(vii) $E_{9}^{*}: d(u)=d(v)=3$;

(viii) $E_{7}\left(\right.$ or $\left.E_{12}^{*}\right): d(u)=3$ and $d(v)=4$.

In terms of further calculating, we check that $|V(S P[n])|=49 n+6$ and $|E(S P[n])|=54 n+5$. More specifically, we deduce $\left|E_{3}\right|=\left|E_{2}^{*}\right|=2 n+1,\left|E_{3}^{*}\right|=9 n+1$, $\left|E_{5} \cap E_{4}^{*}\right|=\left|E_{7}\right|=\left|E_{12}^{*}\right|=n,\left|E_{4} \cap E_{4}^{*}\right|=5 n+4,\left|E_{6}^{*}\right|=18 n-1$, $\left|E_{8}^{*}\right|=2 n$, and $\left|E_{9}^{*}\right|=16 n$. 
Therefore, by the definition of general sum connectivity and general Randic index, we get

$$
\begin{aligned}
& \chi_{k}(S P[n])=\sum_{e=u v}(d(u)+d(v))^{k} \\
& =\sum_{e=u v \in E_{3}}(d(u)+d(v))^{k} \\
& +\sum_{e=u v \in E_{3}^{*}}(d(u)+d(v))^{k} \\
& +\sum_{e=u v \in E_{5} \cap E_{4}^{*}}(d(u)+d(v))^{k} \\
& +\sum_{e=u v \in E_{4} \cap E_{4}^{*}}(d(u)+d(v))^{k} \\
& +\sum_{e=u v \in E_{6}^{*}}(d(u)+d(v))^{k} \\
& +\sum_{e=u v \in E_{8}^{*}}(d(u)+d(v))^{k} \\
& +\sum_{e=u v \in E_{9}^{*}}(d(u)+d(v))^{k} \\
& +\sum_{e=u v \in E_{7}}(d(u)+d(v))^{k} \\
& =(2 n+1) 3^{k}+(9 n+1) 4^{k}+n \cdot 5^{k} \\
& +(5 n+4) 4^{k}+(18 n-1) 5^{k}+2 n \cdot 6^{k} \\
& +16 n \cdot 6^{k}+n \cdot 7^{k} \\
& =(2 n+1) 3^{k}+(14 n+5) 4^{k} \\
& +(19 n-1) 5^{k}+18 n \cdot 6^{k}+n \cdot 7^{k}, \\
& R_{k}(S P[n])=\sum_{e=u v}(d(u) d(v))^{k} \\
& =\sum_{e=u v \in E_{3}}(d(u) d(v))^{k} \\
& +\sum_{e=u v \in E_{3}^{*}}(d(u) d(v))^{k} \\
& +\sum_{e=u v \in E_{5} \cap E_{4}^{*}}(d(u) d(v))^{k} \\
& +\sum_{e=u v \in E_{4} \cap E_{4}^{*}}(d(u) d(v))^{k} \\
& +\sum_{e=u v \in E_{6}^{*}}(d(u) d(v))^{k} \\
& +\sum_{e=u v \in E_{8}^{*}}(d(u) d(v))^{k} \\
& +\sum_{e=u v \in E_{9}^{*}}(d(u) d(v))^{k}
\end{aligned}
$$

$$
\begin{aligned}
& +\sum_{e=u v \in E_{7}}(d(u) d(v))^{k} \\
= & (2 n+1) 2^{k}+(9 n+1) 3^{k}+n \cdot 4^{k} \\
& +(5 n+4) 4^{k}+(18 n-1) 6^{k}+2 n \cdot 8^{k} \\
& +16 n \cdot 9^{k}+n \cdot 12^{k} \\
= & (2 n+1) 2^{k}+(9 n+1) 3^{k}+(6 n+4) 4^{k} \\
& +(18 n-1) 6^{k}+2 n \cdot 8^{k}+16 n \cdot 9^{k}+n \\
& \cdot 12^{k} .
\end{aligned}
$$

Hence, we get the desired conclusion.

In what follows, $S P[n]$ is denoted as the Dox-loaded micelle comprising PEG-PAsp block copolymer with chemically conjugated Dox, and we will not explain this notation again.

Theorem 2. The general harmonic index of $S P[n]$ is

$$
\begin{aligned}
H_{k}(S P[n])= & (2 n+1)\left(\frac{2}{3}\right)^{k}+(14 n+5)\left(\frac{1}{2}\right)^{k} \\
& +(19 n-1)\left(\frac{2}{5}\right)^{k}+18 n\left(\frac{1}{3}\right)^{k} \\
& +n\left(\frac{2}{7}\right)^{k} .
\end{aligned}
$$

Proof. By the definition of general harmonic index and what we have discussed in Theorem 1, we infer

$$
\begin{aligned}
H_{k}(S P[n])= & \sum_{e=u v}\left(\frac{2}{d(u)+d(v)}\right)^{k} \\
= & \sum_{e=u v \in E_{3}}\left(\frac{2}{d(u)+d(v)}\right)^{k} \\
& +\sum_{e=u v \in E_{3}^{*}}\left(\frac{2}{d(u)+d(v)}\right)^{k} \\
& +\sum_{e=u v \in E_{5} \cap E_{4}^{*}}\left(\frac{2}{d(u)+d(v)}\right)^{k} \\
& +\sum_{e=u v \in E_{4} \cap E_{4}^{*}}\left(\frac{2}{d(u)+d(v)}\right)^{k} \\
& +\sum_{e=u v \in E_{6}^{*}}\left(\frac{2}{d(u)+d(v)}\right)^{k}
\end{aligned}
$$




$$
\begin{aligned}
& +\sum_{e=u v \in E_{8}^{*}}\left(\frac{2}{d(u)+d(v)}\right)^{k} \\
& +\sum_{e=u v \in E_{9}^{*}}\left(\frac{2}{d(u)+d(v)}\right)^{k} \\
& +\sum_{e=u v \in E_{7}}\left(\frac{2}{d(u)+d(v)}\right)^{k} \\
& =(2 n+1)\left(\frac{2}{3}\right)^{k}+(9 n+1)\left(\frac{1}{2}\right)^{k} \\
& +n\left(\frac{2}{5}\right)^{k}+(5 n+4)\left(\frac{1}{2}\right)^{k} \\
& +(18 n-1)\left(\frac{2}{5}\right)^{k}+2 n\left(\frac{1}{3}\right)^{k}+16 n \\
& \cdot 6^{k}+n\left(\frac{2}{7}\right)^{k} \\
& =(2 n+1)\left(\frac{2}{3}\right)^{k}+(14 n+5)\left(\frac{1}{2}\right)^{k} \\
& +(19 n-1)\left(\frac{2}{5}\right)^{k}+18 n\left(\frac{1}{3}\right)^{k} \\
& +n\left(\frac{2}{7}\right)^{k} \text {. }
\end{aligned}
$$

We complete the proof. $S P[n]$.

By taking $k=1$ in (18), we yield the harmonic index of

Corollary 3. Consider the following:

$$
H(S P[n])=\frac{2333}{105} n+\frac{83}{30} \text {. }
$$

Using similar fashion, we get the following conclusions on the exact expression of several important indices and polynomials of $S P[n]$.

Theorem 4. The ordinary geometric-arithmetic index of $S P[n]$ is

$$
\begin{aligned}
\mathrm{OGA}_{k}(S P[n])= & (4 n+1)\left(\frac{2 \sqrt{2}}{3}\right)^{k} \\
& +(9 n+1)\left(\frac{2 \sqrt{3}}{4}\right)^{k}+n\left(\frac{4}{5}\right)^{k} \\
& +(21 n+4)+(18 n-1)\left(\frac{2 \sqrt{6}}{5}\right)^{k} \\
& +n\left(\frac{4 \sqrt{3}}{7}\right)^{k}
\end{aligned}
$$

Theorem 5. The generalized version of Zagreb index of $S P[n]$ is

$$
\begin{aligned}
M_{t_{1}, t_{2}}(S P[n])= & (2 n+1)\left(2^{t_{1}}+2^{t_{2}}\right) \\
& +(9 n+1)\left(3^{t_{1}}+3^{t_{2}}\right)+n\left(4^{t_{1}}+4^{t_{2}}\right) \\
& +(5 n+4) 2^{t_{1}+t_{2}+1} \\
& +(18 n-1)\left(2^{t_{1}} 3^{t_{2}}+2^{t_{2}} 3^{t_{1}}\right) \\
& +2 n\left(2^{t_{1}+2 t_{2}}+2^{t_{2}+2 t_{1}}\right)+16 n \\
& \cdot 3^{t_{1}+t_{2}+1}+n\left(3^{t_{1}} 4^{t_{2}}+3^{t_{2}} 4^{t_{1}}\right) .
\end{aligned}
$$

Theorem 6. The first and second Zagreb polynomials of SP $[n]$ are

$$
\begin{aligned}
M_{1}(S P[n], x)= & (2 n+1) x^{3}+(14 n+5) x^{4} \\
& +(19 n-1) x^{5}+18 n x^{6}+n x^{7} \\
M_{2}(S P[n], x)= & (2 n+1) x^{2}+(9 n+1) x^{3} \\
& +(6 n+4) x^{4}+(18 n-1) x^{6} \\
& +2 n x^{8}+16 n x^{9}+n x^{12} .
\end{aligned}
$$

Theorem 7. The third Zagreb index and polynomial of $S P[n]$ are

$$
\begin{aligned}
M_{3}(S P[n])= & 46 n+2, \\
M_{3}(S P[n], x)= & 21 n x+(11 n+1) x^{2}+n x^{3} \\
& +(21 n+4) .
\end{aligned}
$$

Theorem 8. The multiplicative Zagreb indices of $S P[n]$ are

$$
\begin{aligned}
& P M_{1}(S P[n])=3^{2 n+1} 4^{14 n+2} 5^{19 n-1} 6^{18 n} 7^{n}, \\
& P M_{2}(S P[n])=2^{2 n+1} 3^{9 n+1} 4^{6 n+4} 6^{18 n-1} 8^{2 n} 9^{16 n} 12^{n} .
\end{aligned}
$$

Theorem 9. The redefined Zagreb indices of $S P[n]$ are

$$
\begin{aligned}
& \operatorname{Re} Z G_{1}(S P[n])=49 n+6 \text {, } \\
& \operatorname{Re}_{2} G_{2}(S P[n])=\frac{8941}{140} n+\frac{253}{60}, \\
& \operatorname{Re} Z G_{3}(S P[n])=1804 n+52 .
\end{aligned}
$$

\section{Conclusion}

In this paper, inspired by widely used Dox-loaded micelle comprising PEG-PAsp block copolymer with chemically conjugated Dox for anticancer drug study in pharmaceutical engineering applications, we focus on the theoretical analysis of topological indices expression for this molecular structure. By means of edge dividing approaches, we present the exact expression of several important indices, including general sum connectivity, general Randic index, general harmonic 
index, ordinary geometric-arithmetic index, generalized version of Zagreb index, Zagreb polynomials, multiplicative Zagreb indices, and redefined Zagreb indices. The results obtained in our paper illustrate the promising application prospects in chemical and pharmacy engineering.

\section{Conflict of Interests}

The authors declare that there is no conflict of interests regarding the publication of this paper.

\section{Acknowledgment}

The research is partially supported by NSFC (nos. 11401519, 11371328 , and 11471293).

\section{References}

[1] H. J. Wiener, "Structural determination of paraffin boiling points," Journal of the American Chemical Society, vol. 69, no. 1, pp. 17-20, 1947.

[2] A. R. Katritzky, R. Jain, A. Lomaka, R. Petrukhin, U. Maran, and M. Karelson, "Perspective on the relationship between melting points and chemical structure," Crystal Growth \& Design, vol. 1, no. 4, pp. 261-265, 2001.

[3] L. Yan, W. Gao, and J. S. Li, "General harmonic index and general sum connectivity index of polyomino chains and nanotubes," Journal of Computational and Theoretical Nanoscience, vol. 12, no. 10, pp. 3940-3944, 2015.

[4] W. Gao and L. Shi, "Wiener index of gear fan graph and gear wheel graph," Asian Journal of Chemistry, vol. 26, no. 11, pp. 3397-3400, 2014.

[5] W. Gao and W. Wang, "Second atom-bond connectivity index of special chemical molecular structures," Journal of Chemistry, vol. 2014, Article ID 906254, 8 pages, 2014.

[6] W. Gao and W. F. Wang, "The vertex version of weighted wiener number for bicyclic molecular structures," Computational and Mathematical Methods in Medicine, vol. 2015, Article ID 418106, 10 pages, 2015.

[7] J. A. Bondy and U. S. R. Murty, Graph Theory, Spring, Berlin, Germany, 2008.

[8] B. Bollobas and P. Erdos, "Graphs of extremal weights," Ars Combinatoria, vol. 50, pp. 225-233, 1998.

[9] B. Li and W. Liu, "The smallest Randic index for trees," Proceedings-Mathematical Sciences, vol. 123, no. 2, pp. 167-175, 2013.

[10] B. L. Liu and I. Gutman, "Estimating the Zagreb and the general randic indices," MATCH Communications in Mathematical and in Computer Chemistry, vol. 57, no. 3, pp. 617-632, 2007.

[11] B. Zhou and N. Trinajstic, "On general sum-connectivity index," Journal of Mathematical Chemistry, vol. 47, no. 1, pp. 210-218, 2010.

[12] G. H. Shirdel, H. RezaPour, and A. M. Sayadi, "The hyperZagreb index of graph operations," Iranian Journal of Mathematical Chemistry, vol. 4, no. 2, pp. 213-220, 2013.

[13] O. Favaron, M. Maho, and J.-F. Sacle, "Some eigenvalue properties in graphs (conjectures of Graffiti- II)," Discrete Mathematics, vol. 111, no. 1-3, pp. 197-220, 1993.

[14] L. Zhong, "The harmonic index for graphs," Applied Mathematics Letters, vol. 25, no. 3, pp. 561-566, 2012.
[15] R. Wu, Z. Tang, and H. Deng, "A lower bound for the harmonic index of a graph with minimum degree at least two," Filomat, vol. 27, no. 1, pp. 51-55, 2013.

[16] J. X. Liu, “On harmonic index and diameter of graphs," Journal of Applied Mathematics and Physics, vol. 1, no. 3, pp. 5-6, 2013.

[17] M. Eliasi and A. Iranmanesh, "On ordinary generalized geometric-arithmetic index," Applied Mathematics Letters, vol. 24, no. 4, pp. 582-587, 2011.

[18] M. Azari and A. Iranmanesh, "Generalized Zagreb index of graphs," Studia Universitatis Babeş-Bolyai, vol. 56, no. 3, pp. 5970, 2011.

[19] I. Gutman, "Multiplicative Zagreb indices of trees," Bulletin of the Veterinary Institute in Pulawy, vol. 1, pp. 13-19, 2011.

[20] M. Ghorbani and N. Azimi, "Note on multiple Zagreb indices," Iranian Journal of Mathematical Chemistry, vol. 3, pp. 137-143, 2012.

[21] M. Eliasi, A. Iranmanesh, and I. Gutman, "Multiplicative versions of first Zagreb index," MATCH Communications in Mathematical and in Computer Chemistry, vol. 68, no. 1, pp. 217-230, 2012.

[22] K. Xu and K. C. Das, "Trees, unicyclic, and bicyclic graphs extremal with respect to multiplicative sum Zagreb index," MATCH Communications in Mathematical and in Computer Chemistry, vol. 68, no. 1, pp. 257-272, 2012.

[23] M. R. Farahani, "Multiplicative versions of Zagreb indices of TUSC4C8(S)," Journal of Chemistry and Materials Research, vol. 2, pp. 67-70, 2015.

[24] P. S. Ranjini, V. Lokesha, and A. Usha, "Relation between phenylene and hexagonal squeeze using harmonic index," International Journal of Graph Theory, vol. 1, pp. 116-121, 2013.

[25] N. T. M. Hai and P. Broekmann, "Smart hybrid polymers for advanced damascene electroplating: combination of superfill and leveling properties," ChemElectroChem, vol. 2, no. 8, pp. 1096-1099, 2015.

[26] N. do Nascimento Marques, A. M. da Silva Maia, and R. de Carvalho Balaban, "Development of dual-sensitive smart polymers by grafting chitosan with poly ( $N$-isopropylacrylamide): an overview," Polímeros, vol. 25, no. 3, pp. 237-246, 2015.

[27] A. Kroning, A. Furchner, D. Aulich et al., "In situ infrared ellipsometry for protein adsorption studies on ultrathin smart polymer brushes in aqueous environment," ACS Applied Materials \& Interfaces, vol. 7, no. 23, pp. 12430-12439, 2015.

[28] A. Chonkar, U. Nayak, and N. Udupa, "Smart polymers in nasal drug delivery," Indian Journal of Pharmaceutical Sciences, vol. 77, no. 4, pp. 367-375, 2015.

[29] M. Hrubý, S. K. Filippov, and P. Štěpánek, "Smart polymers in drug delivery systems on crossroads: which way deserves following," European Polymer Journal, vol. 65, pp. 82-97, 2015.

[30] A. Duro-Castano, J. Movellan, and M. J. Vicent, "Smart branched polymer drug conjugates as nano-sized drug delivery systems," Biomaterials Science, vol. 3, no. 10, pp. 1321-1334, 2015.

[31] J. Khandare and M. Calderón, "Dendritic polymers for smart drug delivery applications," Nanoscale, vol. 7, no. 9, pp. 38063807, 2015.

[32] K. Shanthi, K. Vimala, D. Gopi, and S. Kannan, "Fabrication of a $\mathrm{pH}$ responsive DOX conjugated PEGylated palladium nanoparticle mediated drug delivery system: an in vitro and in vivo evaluation," RSC Advances, vol. 5, no. 56, pp. 44998-45014, 2015.

[33] K. Osada, R. J. Christie, and K. Kataoka, "Polymeric micelles from poly(ethylene glycol)-poly(amino acid) block copolymer 
for drug and gene delivery," Journal of the Royal Society Interface, vol. 6, no. 3, pp. S325-S339, 2009.

[34] N. Nishiyama and K. Kataoka, "Polymeric micelle drug carrier systems: PEG-PAsp(Dox) and second generation of micellar drugs," in Polymer Drugs in the Clinical Stage, vol. 519 of Advances in Experimental Medicine and Biology, pp. 155-177, Springer, 2003.

[35] A. M. Butt, M. C. Iqbal, M. Amin, and H. Katas, "Synergistic effect of $\mathrm{pH}$-responsive folate-functionalized poloxamer 407TPGS-mixed micelles on targeted delivery of anticancer drugs," International Journal of Nanomedicine, vol. 10, pp. 1321-1334, 2015. 

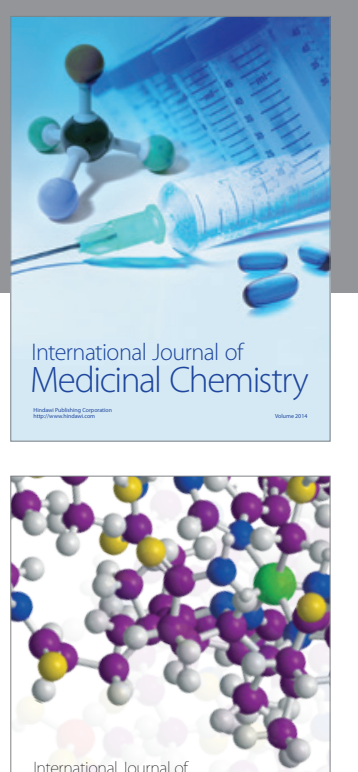

Carbohydrate Chemistry

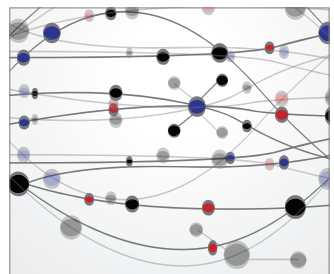

The Scientific World Journal
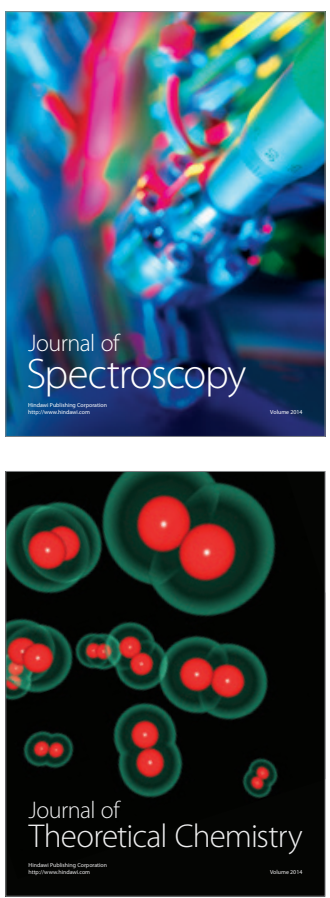
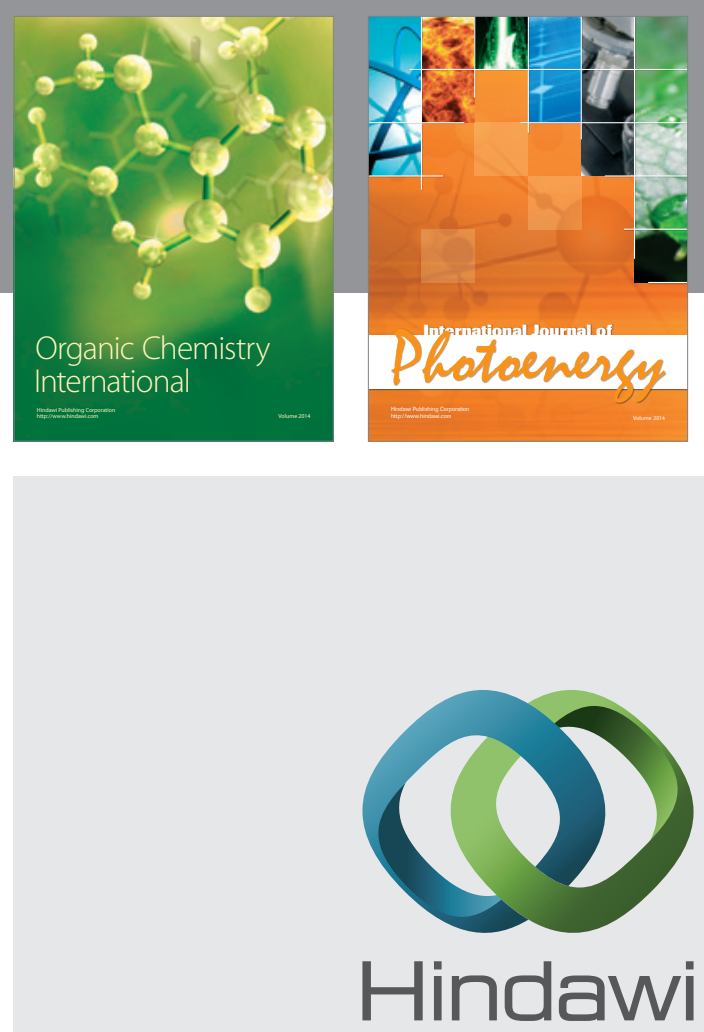

Submit your manuscripts at

http://www.hindawi.com

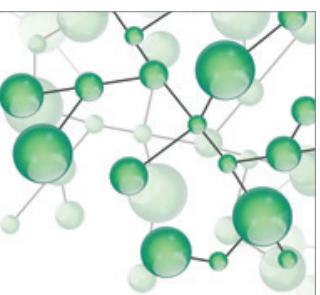

International Journal of

Inorganic Chemistry

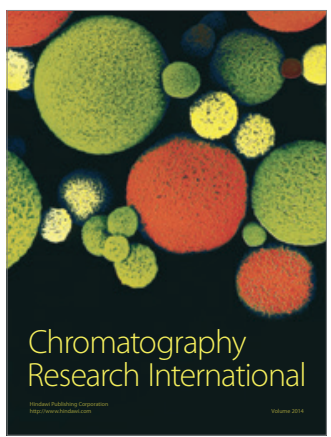

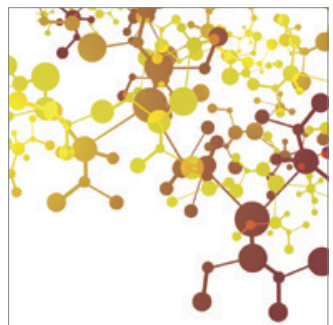

Applied Chemistry
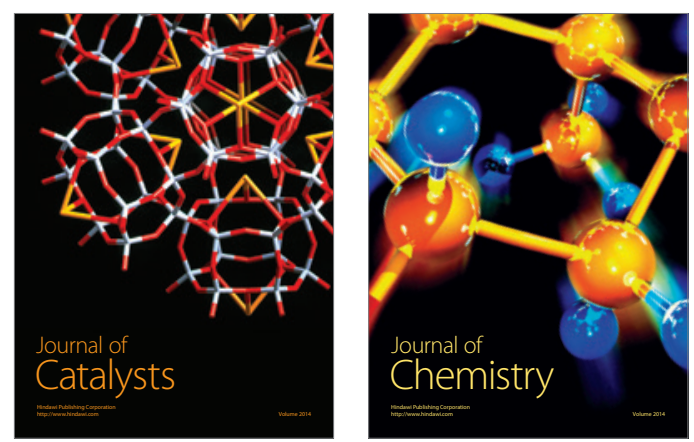
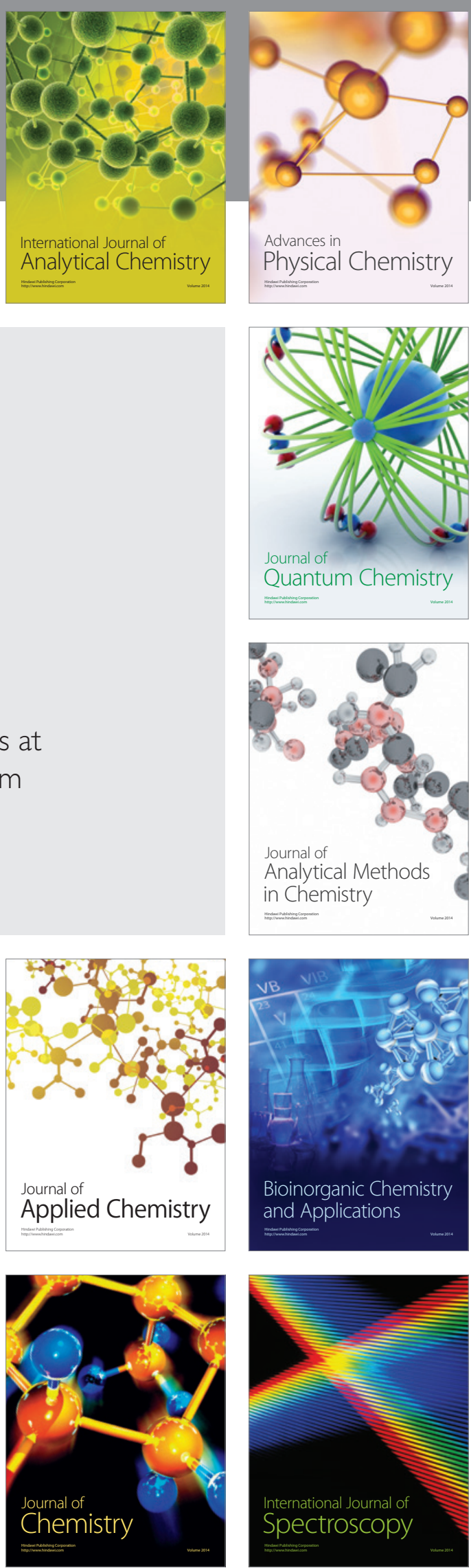